\title{
Use of contralateral spinal accessory nerve for ipsilateral suprascapular neurotization in global brachial plexus injury: a new technique
}

\author{
Prem Singh Bhandari, MS, MCh, ${ }^{1}$ and Prabal Deb, MD² \\ 'Department of Plastic and Reconstructive Surgery, Command Hospital, Udhampur, Jammu \& Kashmir; and 2Department of \\ Neuropathology, Army Hospital Referral \& Research, Delhi, India
}

\begin{abstract}
Nerve transfer between the spinal accessory nerve (SAN) and the suprascapular nerve (SSN) is a standard technique in shoulder reanimation. In cases of global brachial plexus injury, donor nerves are few and at times severely traumatized owing to extensive traction forces. This precludes the application of standard nerve transfer techniques. The authors offer the use of the contralateral SAN as an additional option in the reinnervation of an injured SSN in such circumstances. To the best of their knowledge, this is the first successful attempt of this technique to be reported in the literature. http://thejns.org/doi/abs/10.3171/2015.4.SPINE15108
\end{abstract}

KEY WORDS contralateral spinal accessory nerve; global brachial plexus injury; ipsilateral suprascapular neurotization; technique

$\mathrm{T}$ HE spinal accessory nerve (SAN), being predominantly a motor nerve, is widely used in ipsilateral nerve transfer to the suprascapular nerve (SSN). In the conventional approach, the SAN is dissected through a supraclavicular incision, is divided retroclavicularly, and is directly transferred to the SSN. This approach carries a risk of partial denervation of the upper trapezius muscle. A nerve transfer performed through a posterior approach preserves the proximal branches to the upper trapezius and allows nerve transfer close to the target muscles. ${ }^{1,2}$ An ipsilateral phrenic nerve is another effective donor nerve but its is known to result in a transient decrease in pulmonary function tests. ${ }^{4}$ However, both the SAN and the phrenic nerve are vulnerable to injury from severe traction forces in the head and neck regions, as occurs at times in global brachial plexus injury. Therefore neurotization of the SSN becomes a real challenge. In this paper we offer the use of the contralateral SAN as an additional option when no local transfers are available.

\section{Case Report}

History

A 29 -year-old man sustained a total brachial plexus injury in his right upper extremity during a motorcycle accident and reported to a tertiary care center 4 months later.

\section{Examination}

Examination revealed a flail and anesthetic limb with drooped shoulder and a positive Horner sign. The ipsilateral trapezius muscle was paralyzed. The sternocleidomastoid muscle failed to contract when the patient turned his neck to the contralateral side. A plain radiograph of the chest revealed a healing fracture of the right clavicle with a raised dome of the ipsilateral diaphragm. An anteroposterior radiograph of the cervical spine revealed a fracture of the transverse process of C-7. A 3D MRI study revealed pseudomeningoceles at the C-7, C-8, and T-1 root levels. Electromyography revealed denervation of paraspinal muscles. The contralateral extremity was normal, with the power of the trapezius muscle recorded as M5. Surgical reconstruction was planned, and various treatment options were discussed with the patient.

\section{Intraoperative Findings}

On exploration of the supraclavicular brachial plexus, at 4 months after the injury, an extensive fibrosis was noticed in the scalene triangle and adjoining area. No nerve elements were found in the scar tissue. The phrenic nerve could not be located on the surface of the scarred anterior scalene muscle. The SSN, emerging from the distally retracted part of the plexus, failed to respond to an electric 


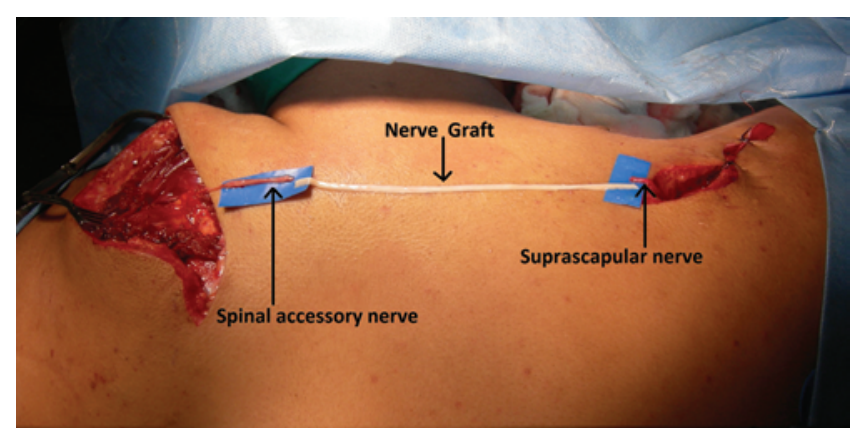

FIG. 1. Intraoperative photograph showing the 13.5 -cm-long sural nerve graft interposed between the ends of the SAN and SSN. Figure is available in color online only.

stimulus. The SAN was thin and fibrotic and showed a weak response to a stimulus of $2 \mathrm{~mA}$.

\section{Operative Procedure}

The patient was placed in a semilateral position with head of the operating table raised $40^{\circ}$. Through a transverse incision made parallel to the spine of scapula, the distal part of the SSN was dissected up to the suprascapular notch. The SSN, which measured $7.5 \mathrm{~cm}$ from the upper trunk to the suprascapular notch, was then divided at its takeoff point from the upper trunk and delivered through the scapular incision. At this stage, the patient was placed in the prone position. Through another incision over the contralateral scapular spine, the SAN was dissected free, with care taken to preserve the branches to the upper and middle parts of the trapezius muscle. The distal vertical course of the SAN was dissected along the medial border of scapula. The nerve was divided as it started thinning off, approximately $5 \mathrm{~cm}$ from the superior angle of scapula, and was delivered to the surface. The distance between the SAN and SSN measured $12 \mathrm{~cm}$. A $13.5-\mathrm{cm}-$ long sural nerve graft, tunneled subcutaneously, was interposed between the ends of the SAN and SSN (Fig. 1). A third vertical midline incision helped in the retrieval of the nerve graft. Figure 2 shows a schematic representation of this technique. Nerve coaptations were carried out with 10-0 nylon suture under a surgical microscope. Skin incisions were closed with multiple metal staples. Additional nerve transfer for restoration of elbow flexion consisted of transfer of the third, fourth, and fifth intercostal nerves to the musculocutaneous nerve.

\section{Postoperative Course}

Postoperatively, the operated arm was strapped to the patient's chest for a period of 3 weeks, following which stretching exercises and electrical stimulation were initiated. The patient was followed up at monthly intervals during the first 6 months. Beginning at 20 weeks after the operation, 8 weekly electromyographic examinations were performed on the infraspinatus muscle. The muscle power of the trapezius was examined at 6 months after reconstruction. The first electrophysiological sign of reinnervation of the infraspinatus muscle appeared 48 weeks after the operation, whereas initial evidence of restoration of shoulder abduction was noticed 52 weeks after the operation. Two years (24 months) postoperatively, electromyography revealed polyphasic motor unit potentials in the infraspinatus muscle. Clinically, the patient had a stable shoulder with $26^{\circ}$ of active abduction and $15^{\circ}$ of external rotation (Fig. 3A and 3B). Shoulder abduction was synchronous with the elevation of the contralateral shoulder (Fig. 3C).

\section{Discussion}

Restoration of shoulder stability and function constitutes an important goal in the rehabilitation of patients with global brachial plexus injuries. Nerve transfer between the SAN and SSN helps in achieving these goals. In
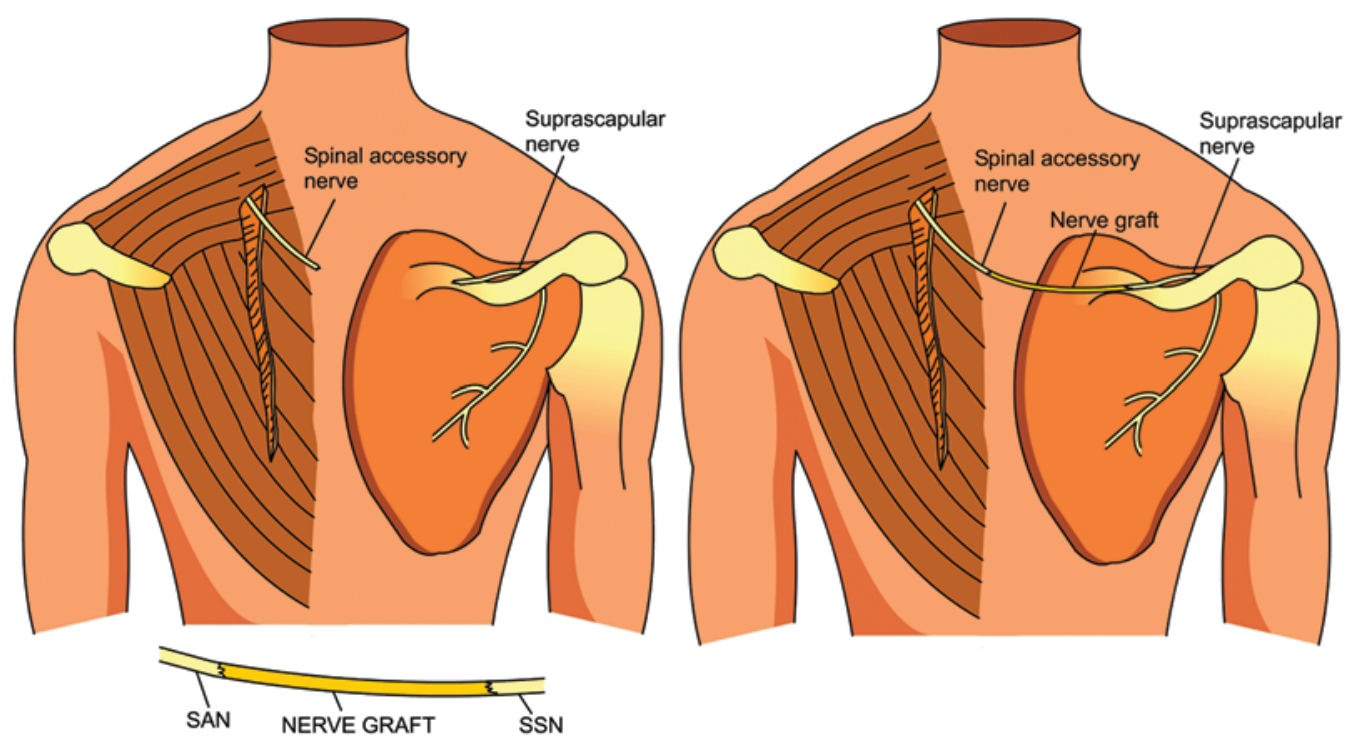

FIG. 2. Schematic representation of our technique. Copyright Prabal Deb. Published with permission. Figure is available in color online only. 

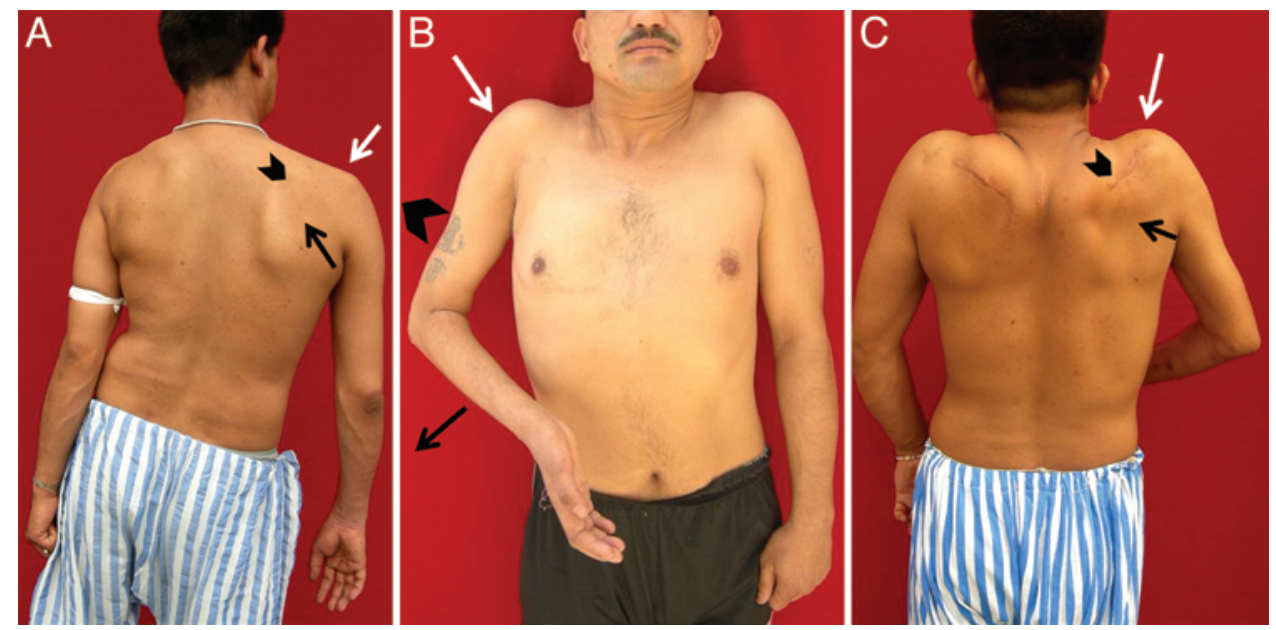

FIG. 3. A: Preoperative photograph showing a flail limb with drooping of the right shoulder (white arrow) and paralyzed supraspinatus (black arrowhead) and infraspinatus muscles (black arrow). B: Restoration of a stable right shoulder (white arrow) with $26^{\circ}$ of active abduction (black arrowhead) and $15^{\circ}$ of external rotation (black arrow). C: Abduction of reconstructed right shoulder (white arrow) was synchronous with elevation of the contralateral shoulder. In addition, the bulk of the supraspinatus (black arrowhead) and infraspinatus muscles (black arrow) has been restored. Figure is available in color online only.

cases of severe grades of brachial plexus injury, root avulsions are common and at times associated with an extended injury to the potential donor nerves (e.g., phrenic nerve and SAN). Under these circumstances, the contralateral C-7 spinal nerve has been transferred to the SSN and axillary nerves to regain shoulder function. ${ }^{6}$ In a recent study consisting of 13 cases, Sammer et al. noted functional outcomes following hemicontralateral C-7 nerve transfer to the axillary nerve and SSN. ${ }^{3}$ The donor nerve was transferred end-to-end to the axillary nerve and/or the SSN with the use of reversed nonvascularized sural-nerve cable grafts. Although most of the patients demonstrated electromyographic evidence of motor reinnervation of one or more of the target muscles, only 3 patients achieved functional (M3 or greater) shoulder abduction. None of the patients regained active external rotation of the shoulder.

In the present case, no viable root stumps were available. At the same time, potential donor nerves (SAN, phrenic nerves) were also injured. Our previous experience with histomorphometry has shown an adequacy of myelinated axons in the distal part of the SAN. ${ }^{1}$ A search of the extant literature on the subject revealed that contralateral SAN might be considered for ipsilateral neurotization of the suprascapular nerve, using a posterior approach. ${ }^{5}$ This encouraged us to use the contralateral SAN, lengthened with a graft, to reinnervate the injured SSN. At 24 months' follow-up our patient had regained $26^{\circ}$ of active shoulder abduction and $15^{\circ}$ of external rotation. This leads us to believe that in severe grades of brachial plexus injury with associated injuries to the ipsilateral SAN and phrenic nerve, use of the contralateral SAN is a viable option in restoration of shoulder function. However, further experience needs to be gained from a larger number of cases before this can be accepted as a standard technique.

\section{References}

1. Bhandari PS, Deb P: Dorsal approach in transfer of the distal spinal accessory nerve into the suprascapular nerve: histomorphometric analysis and clinical results in 14 cases of upper brachial plexus injuries. J Hand Surg Am 36:1182-1190, 2011

2. Bhandari PS, Deb P: Posterior approach for both spinal accessory nerve to suprascapular nerve and triceps branch to axillary nerve for upper plexus injuries. J Hand Surg Am 38:168-172, 2013

3. Sammer DM, Kircher MF, Bishop AT, Spinner RJ, Shin AY: Hemi-contralateral C7 transfer in traumatic brachial plexus injuries: outcomes and complications. J Bone Joint Surg Am 94:131-137, 2012

4. Siqueira MG, Martins RS: Phrenic nerve transfer in the restoration of elbow flexion in brachial plexus avulsion injuries: how effective and safe is it? Neurosurgery 65 (4 Suppl):A125-A131, 2009

5. Tubbs RS, Mortazavi MM, Shoja MM, Loukas M, CohenGadol AA: Contralateral spinal accessory nerve for ipsilateral neurotization of branches of the brachial plexus: a cadaveric feasibility study. J Neurosurg 114:1538-1540, 2011

6. Zhang CG, Gu YD: Contralateral C7 nerve transfer - Our experiences over past 25 years. J Brachial Plex Peripher Nerve Inj 6:10, 2011

\section{Disclosure}

The authors report no conflict of interest concerning the materials or methods used in this study or the findings specified in this paper.

\section{Author Contributions}

Conception and design: Bhandari. Acquisition of data: Bhandari. Analysis and interpretation of data: Deb. Drafting the article: Deb. Critically revising the article: Deb. Reviewed submitted version of manuscript: Bhandari. Approved the final version of the manuscript on behalf of both authors: Deb. Administrative/technical/material support: Bhandari. Study supervision: Bhandari.

\section{Correspondence}

Prabal Deb, Department of Pathology, Army Hospital (Research \& Referral), Dhaula Kuan, New Delhi 110010, India. email: debprabal@gmail.com. 